\title{
Directional bending wave propagation in periodically perforated plates
}

\author{
Andreassen, Erik; Manktelow, Kevin ; Ruzzene, Massimo
}

Published in:

Journal of Sound and Vibration

Link to article, DOI:

10.1016/j.jsv.2014.09.035

Publication date:

2015

Link back to DTU Orbit

Citation (APA):

Andreassen, E., Manktelow, K., \& Ruzzene, M. (2015). Directional bending wave propagation in periodically perforated plates. Journal of Sound and Vibration, 335, 187-203. https://doi.org/10.1016/j.jsv.2014.09.035

\section{General rights}

Copyright and moral rights for the publications made accessible in the public portal are retained by the authors and/or other copyright owners and it is a condition of accessing publications that users recognise and abide by the legal requirements associated with these rights.

- Users may download and print one copy of any publication from the public portal for the purpose of private study or research

- You may not further distribute the material or use it for any profit-making activity or commercial gain

- You may freely distribute the URL identifying the publication in the public portal

If you believe that this document breaches copyright please contact us providing details, and we will remove access to the work immediately and investigate your claim 


\title{
Directional bending wave propagation in periodically perforated plates
}

\author{
Erik Andreassen ${ }^{\mathrm{a}, *}$, Kevin Manktelow ${ }^{\mathrm{b}}$, Massimo Ruzzene ${ }^{\mathrm{b}}$ \\ ${ }^{a}$ Department of Mechanical Engineering, Technical University of Denmark, Nils Koppels \\ Allé, Building 404, Denmark \\ ${ }^{b}$ School of Aerospace Engineering, Georgia Institute of Technology, Atlanta, GA 30332
}

\begin{abstract}
We report on the investigation of wave propagation in a periodically perforated plate. A unit cell with double-C perforations is selected as a test article suitable to investigate two-dimensional dispersion characteristics, group velocities, and internal resonances. A numerical model, formulated using Mindlin plate elements, is developed to predict relevant wave characteristics such as dispersion, and group velocity variation as a function of frequency and direction of propagation. Experimental tests are conducted through a scanning laser vibrometer, which provides full wave field information. The analysis of time domain wave field images allows the assessment of plate dispersion, and the comparison with numerical predictions. The obtained results show the predictive ability of the considered numerical approach and illustrate how the considered plate configuration could be used as the basis for the design of phononic waveguides with directional and internal resonant characteristics.
\end{abstract}

Keywords: Perforated plate, Double-C resonator, Phononic plate,

*E-mail: erand@mek.dtu.dk 
Scanning laser vibrometer.

\section{Introduction}

Recently, materials and structures with periodic modulations in their physical properties have attracted significant attention due to their unusual electromagnetic, acoustic and elastic properties. Such properties lead to unique wave propagation characteristics that can be exploited for the design of waveguides, filters, focusing devices, as well as structural assemblies with negative inertial properties and refractive characteristics. Thus, periodic structural configurations often form the basis for the design of metamaterials, and photonic and phononic crystals $[1,2,3,4]$.

Several configurations of acoustic metamaterials and phononic crystals have been proposed in recent years. These designs have illustrated the ability to tailor equivalent material properties, and to achieve interesting dynamic

phenomena such as negative refraction [5, 6, 7, 8], wave beaming [9], and bandgaps $[10,11,12,13]$. Among the configurations proposed, the use of plate structures as propagation media is an attractive solution due to the existence of several theories to support the formulation of numerical models, and the ease of experimentation provided by optical measurement systems such as scanning laser vibrometers and interferometers. For these reasons, a number of recent studies have investigated elastic plates with periodic inclusions and voids, microstructures, and sources of impedance mismatch such as surface mounted stubs $[14,15,16]$ to generate phononic plates and elastoacoustic metamaterials $[17,18]$. In particular, the work of $[12,8,6,7]$ focuses on flexural (transverse) waves in periodically perforated plates. 
The objective of this paper is to conduct the numerical and experimental investigation of transverse wave motion in a periodically perforated plate. Transversely polarized plate wave modes correspond to those modes characterizing the low frequency/long wavelength Lamb wave spectrum and often specifically to the first asymmetric mode $\left(A_{0}\right)[19]$. At low enough frequencies this mode can be well approximated by plate theories such as Kirchhoff's and Mindlin's [20]. The configuration of the unit cell of the periodic assembly consists of double-C perforations following Guenneau et al. [5], which are also reminiscent of electromagnetic metamaterial designs [21]. This specific configuration is interesting for a number of reasons. First, the periodic array of perforations is expected to provide the plate with partial and full frequency bandgaps, i.e. frequency ranges within which wave propagation is impeded either omnidirectionally or only for a specific range of directions. Such bandgaps may be the result of Bragg scattering, or may be produced by local resonances within the unit cell [22]. In addition, the non-isotropic unit cell design is also expected to lead to an overall, frequency-dependent anisotropy of the plate behavior, and consequently to the onset of wave directionality $[9,23]$.

A numerical model is developed for the perforated plate using rectangular Mindlin plate elements. The model is validated by comparing with the predictions of a solid model. This also highlights the frequency range of accuracy of the formulation. Next, scanning laser vibrometer wave field measurements are conducted to further validate the numerical model and highlight the occurrence of anomalous wave motion, directional behavior and local resonances occurring within the considered plate. Specifically, wave field data 
are recorded over the entire plate to compute dispersion relations through the application of multidimensional (2D and 3D) Fourier transforms (FTs) [24], to visualize the shape of propagating wavefronts, and compare them with estimations based on dispersion surfaces and group velocity computations based on unit cell analysis and the application of Bloch theorem [25].

The paper is organized in four sections, including this introduction. Section 2 briefly describes the considered plate configuration, and presents the formulation of the numerical model for the evaluation of the dispersion characteristics of the plate. Section 3 illustrates the experimental setup, the procedures, and the signal processing methodologies used for the evaluation of the wave properties based on experimental data. Finally, Section 4 summarizes the main findings of the work and provides recommendations for future investigations.

\section{Numerical analysis of wave motion}

\subsection{Configuration}

The considered periodic plate and unit cell configuration are shown in Fig. 1. This configuration is expected to be characterized by higher equivalent stiffness along the longitudinal direction, and lower stiffness along the vertical direction, and thus appears as inherently anisotropic. Stiffness directionality has previously been described as one essential mechanism for the generation of frequency "caustics" [26, 27], which in turn are responsible for the angular concentration of wave vectors at specific frequencies and their directional interference. In addition, the double-C perforations naturally define a resonating unit within the unit cell, which leads to localized modes and 

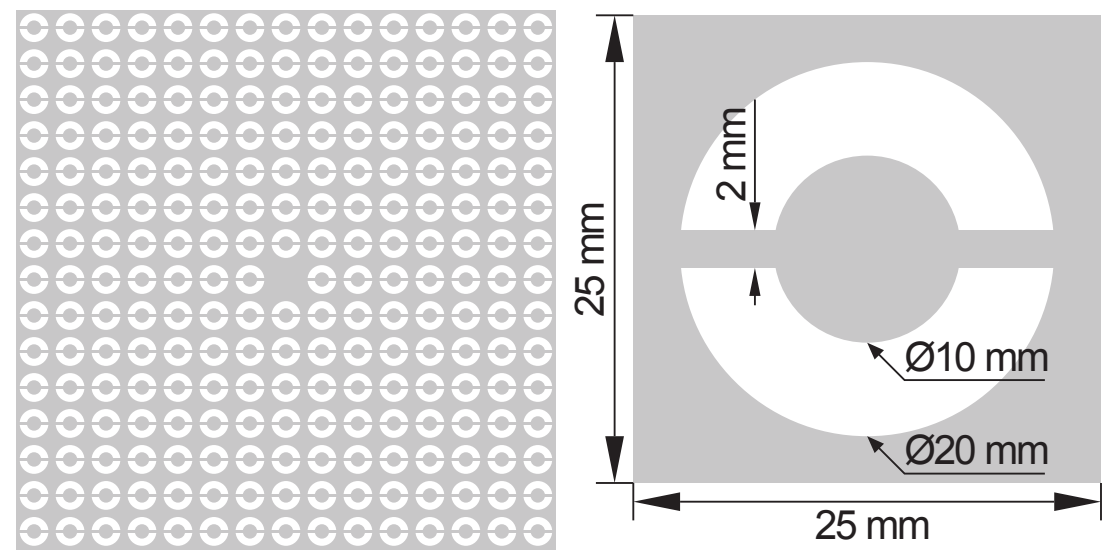

Figure 1: (a) Perforated plate, and (b) unit cell geometry and dimensions. The thickness of the plate is $1 \mathrm{~mm}$.

the generation of internal resonance frequency bandgaps [22, 28, 29]. The considered plate arrangement therefore appears as a convenient configuration for the numerical and experimental investigation of wave directionality and resonant bandgaps, which are two of the predominant features of elastic metamaterials.

\subsection{Approach}

A numerical model of the plate in Fig. 1 is developed to estimate its dynamic behavior, and in particular to predict its dispersion properties and corresponding wave velocities. The model is a finite element (FE) based discretization of the structure by means of four-node Mindlin plate (MP) elements [20]. MP elements are here used as a best compromise between the ability to capture high frequency Lamb wave modes that are typically too coarsely approximated by Kirchoff plate formulations, and the computational cost that is associated with the three-dimensional (3D) discretization of the 
plate. Remark, for at least the lower part of the frequency range considered in this paper Kirchhoff plate formulation would have been just as good, but the presented Mindlin formulation is directly applicable to higher frequencies and thicker plates. Focus is placed on the out-of-plane motion (bending) of the plate, which can be conveniently measured through the available scanning laser vibrometer system. Results in the upcoming subsections illustrate how the considered model is effective at capturing the low frequency predominantly transversely polarized, or antisymmetric, Lamb modes of the plate, which are of interest to the present study [19].

\subsection{Governing equations}

The out-of-plane dynamic behavior of the plate considered herein can be described by the following coupled set of governing differential equations [30]:

$$
\begin{gathered}
\boldsymbol{\nabla}^{T} \mathbf{G}(\boldsymbol{\nabla} w-\boldsymbol{\Theta})=\rho h \frac{\partial^{2} w}{\partial t^{2}} \\
\boldsymbol{\partial}^{T} \mathbf{D} \boldsymbol{\partial} \boldsymbol{\Theta}+\mathbf{G}(\boldsymbol{\nabla} w-\boldsymbol{\Theta})=\frac{\rho h^{3}}{12} \frac{\partial^{2} \boldsymbol{\Theta}}{\partial t^{2}}
\end{gathered}
$$

where $w(\mathbf{x}, t)$ denotes the out-of-plane deflection of a point of the mid-surface at location $\mathbf{x}=[x, y]^{T}, \boldsymbol{\Theta}=\left[\theta_{x}, \theta_{y}\right]^{T}$ is a vector containing the the two rotations of a material line normal to the mid-surface about the two in-plane axes, $\rho$ is the material density, while $h$ is the plate thickness. Also, $\mathbf{D}=\mathbf{D}(\mathbf{x})$ and $\mathbf{G}=\mathbf{G}(\mathbf{x})$ are the plate constitutive matrices. At a given point in the plate material, which is locally isotropic, these are:

$$
\mathbf{D}=\frac{E h^{3}}{12\left(1-\nu^{2}\right)}\left[\begin{array}{ccc}
1 & \nu & 0 \\
\nu & 1 & 0 \\
0 & 0 & \frac{(1-\nu)}{2}
\end{array}\right]
$$


and

$$
\mathbf{G}=\frac{E}{2(1+\nu)}\left[\begin{array}{cc}
\frac{5}{6} h & 0 \\
0 & \frac{5}{6} h
\end{array}\right]
$$

Finally, the differential operator $\boldsymbol{\partial}$ in Eq. (1) is defined as:

$$
\boldsymbol{\partial}=\left[\begin{array}{ll}
1 & 0 \\
0 & 0 \\
0 & 1
\end{array}\right] \frac{\partial}{\partial x}+\left[\begin{array}{ll}
0 & 0 \\
0 & 1 \\
1 & 0
\end{array}\right] \frac{\partial}{\partial y}=\boldsymbol{\alpha}_{1} \frac{\partial}{\partial x}+\boldsymbol{\alpha}_{2} \frac{\partial}{\partial y}
$$

The objective is to investigate the propagation of plane waves associated with the bending motion of the periodic plate. Therefore, solutions in the form of a Bloch-expansion [25]:

$$
\left[\begin{array}{l}
w(\mathbf{x}, t) \\
\boldsymbol{\Theta}(\mathbf{x}, t)
\end{array}\right]=\left[\begin{array}{c}
\tilde{w}(\mathbf{x}) \\
\tilde{\Theta}(\mathbf{x})
\end{array}\right] e^{i(\mathbf{k} \cdot \mathbf{x}+\omega t)}
$$

where $\tilde{w}$ and $\tilde{\boldsymbol{\Theta}}$ are periodic functions whose period coincides with the unit cell, $\omega$ is the angular frequency, and $\mathbf{k}=\left[k_{x}, k_{y}\right]^{T}$ is the wave vector.

Equation (1) can be rewritten in terms of the periodic fields to give:

$$
\nabla_{k}^{T} \mathbf{G}\left(\nabla_{k} \tilde{w}-\tilde{\boldsymbol{\Theta}}\right)=-\omega^{2} \rho h \tilde{w}
$$

where a new operator $\nabla_{k}$ is defined as

$$
\boldsymbol{\nabla}_{k}=\boldsymbol{\nabla}+i \mathbf{k}=\boldsymbol{\beta}_{1}\left(\frac{\partial}{\partial x}+i k_{x}\right)+\boldsymbol{\beta}_{2}\left(\frac{\partial}{\partial y}+i k_{y}\right)
$$

where $\boldsymbol{\beta}_{1}=(1,0)^{T}$, and $\boldsymbol{\beta}_{2}=(0,1)^{T}$.

Similarly, Eq. (2) can be rewritten as

$$
\boldsymbol{\partial}_{k}^{T} \mathbf{D} \boldsymbol{\partial}_{k} \tilde{\boldsymbol{\Theta}}+\mathbf{G}\left(\nabla_{k}^{T} \tilde{w}-\tilde{\boldsymbol{\Theta}}\right)=-\omega^{2} \frac{\rho h^{3}}{12} \tilde{\boldsymbol{\Theta}}
$$

where the operator $\boldsymbol{\partial}_{k}$ is defined as

$$
\boldsymbol{\partial}_{k}=\boldsymbol{\alpha}_{1}\left(\frac{\partial}{\partial x}+i k_{x}\right)+\boldsymbol{\alpha}_{2}\left(\frac{\partial}{\partial y}+i k_{y}\right)
$$




\subsection{Finite element discretization}

Equations (7) and (9) can be discretized using a standard Galerkin approach. The result can be cast in the same form as the in-plane wave propagation problem described in [31]. The mesh consists of 6560 square-shaped Mindlin plate (MP) elements with side lengths of $0.25 \mathrm{~mm}$. The mesh is selected upon a convergence study conducted on the predicted dispersion relations evaluated over the frequency range of interest in this study, as specified below. The smallest wavelength of interest is around half the unit cell width, which means the chosen discretization should be fine enough to resolve it. Also the mesh is refined enough to produce an acceptable description of the internal geometry of the cell perforations, which cannot be perfectly resolved by the considered the mesh, as illustrated in Fig. 2. However, the use of square-shaped elements simplifies the meshing procedure and the model formulation as a whole, and represents a natural choice when considering a possible future extension of this work to design optimization of the unit cell. Furthermore, MP elements provide acceptable approximation of the lower frequency wave modes of the plate with a computational cost that enables the exploration of the full dispersion surfaces and the estimation of wave velocities, which is one of the objectives of this study. In order to avoid numerical inaccuracies due to shear locking, selective reduced integration of shear deformation terms has been used when computing element stiffness matrices.

\subsection{Estimation of dispersion properties}

The discretized equation for a unit cell can be generally expressed as:

$$
\left(\mathbf{K}-\omega^{2} \mathbf{M}\right) \mathbf{u}=\mathbf{0}
$$




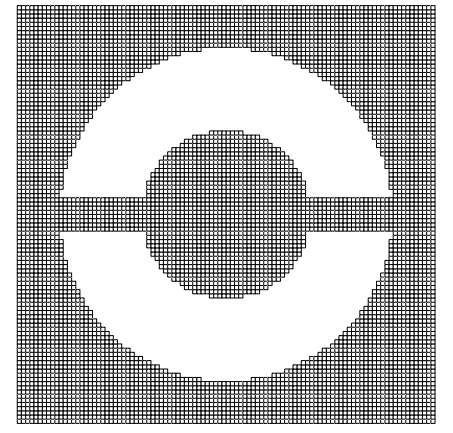

Figure 2: The mesh used to discretize the unit cell consists of 6560 squareshaped elements.

where

$$
\mathbf{K}=\mathbf{K}_{0}-i\left(k_{x} \mathbf{K}_{1}+k_{y} \mathbf{K}_{2}\right)+k_{x}^{2} \mathbf{K}_{3}+k_{x} k_{y} \mathbf{K}_{4}+k_{y}^{2} \mathbf{K}_{5}
$$

and $\mathbf{u}$ is the vector of the generalized nodal displacements of the unit cell. Expressions for the matrices above can be found in Appendix A. Solution of Eq. (11) in terms of $\omega$ for an assigned wave vector $\mathbf{k}$ leads to the dispersion relation of the periodic plate. As customary, the dispersion relations are evaluated and plotted for a wave vector varying along the boundary of the irreducible Brillouin zone, which leads to the band diagram representation. The boundary of the irreducible Brillouin zone is the line O-A-B-C-O in Fig. 3.

The procedure above is applied to compute the band diagram for the considered periodic domain, which is shown in Fig. 4. The diagram is estimated by considering the mesh presented in Fig. 2, which corresponds to the discretization of the thinnest member of the assembly being discretized by eight elements, an even number that helps preserving the symmetry of the geometry. This mesh provides an accurate estimation of the band diagram 


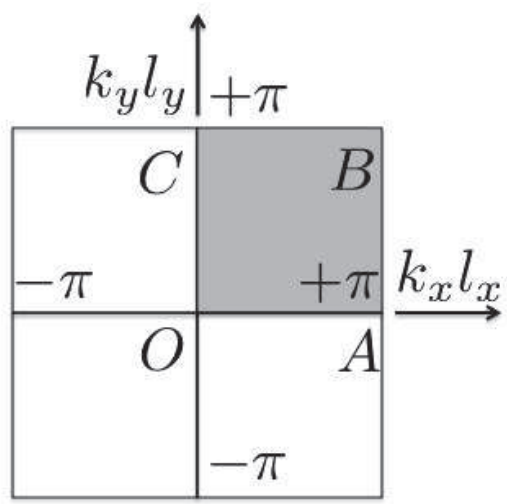

Figure 3: Illustration of the first Brillouin zone and of the irreducible Brillouin zone (gray area). The parameters $l_{x}$ and $l_{y}$ are the unit cell's width and height, respectively.

within the considered frequency range. This is verified by comparing the corresponding diagrams with those obtained from a mesh with elements of half the size. Such comparison does not show any noticeable difference, thus the coarser mesh is used for follow on investigations because of computational efficiency.

Figure 4 compares the dispersion branches estimated through the MP elements (red dots), and through a 3D mesh consisting of eight-node solids (open circles) using three elements to discretize the plate thickness. The 3D model also provides the in-plane polarized modes (red circles), which mostly appear independent from the predominantly out-of-plane branches (black circles) in a frequency range up to approximately $40 \mathrm{kHz}$. The comparison confirms that a plate model is suitable for wave propagation characterization in the considered frequency range.

The contour plots of the first four dispersion surfaces corresponding to 


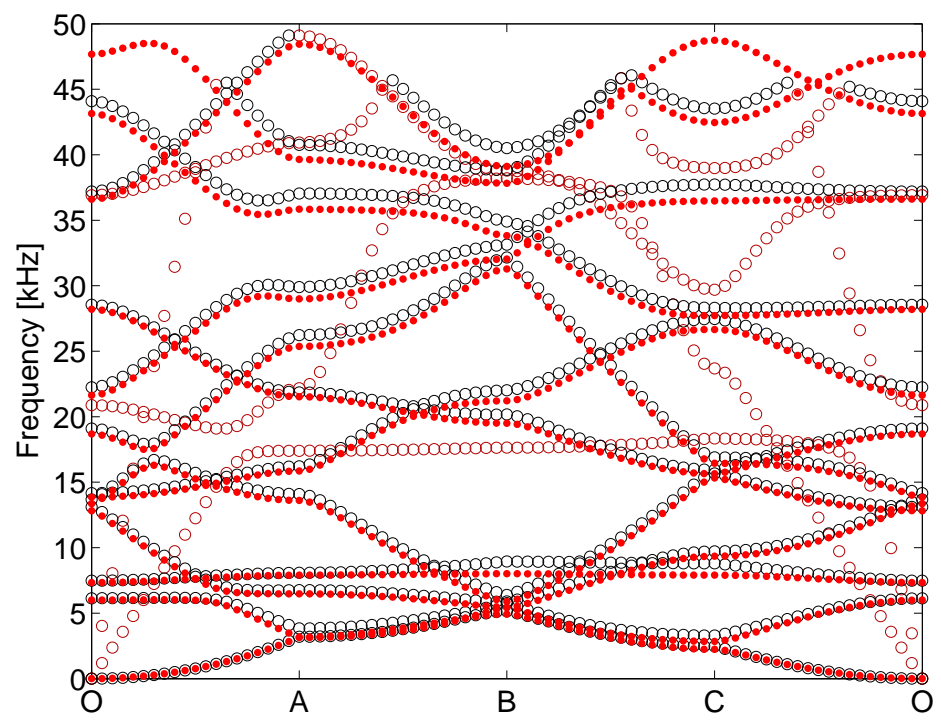

Figure 4: Band diagram: comparison of MP model (red dots) and 3D model predictions showing the decoupling of in-plane (red circles) and out-of-plane (black circles) polarized modes in the considered range of frequencies.

the first four branches in Fig. 4 are shown in Fig. 5, which illustrates the potential for directionality of the considered plate configuration. Namely, the isofrequency contours for the first dispersion surface appear as approximately circular, which suggests a quasi-isotropic behavior in this frequency range. In contrast, the dispersion surfaces for the third and fourth mode in particular appear as mostly aligned along the vertical axis, at frequencies around 7 and $9 \mathrm{kHz}$ respectively. This suggests that directionality along the horizontal direction may occur when wave motion at these frequencies and according to these modes takes place within the plate. Directionality will be further illustrated through the analysis of group velocities, based on the developments presented in the next section. 


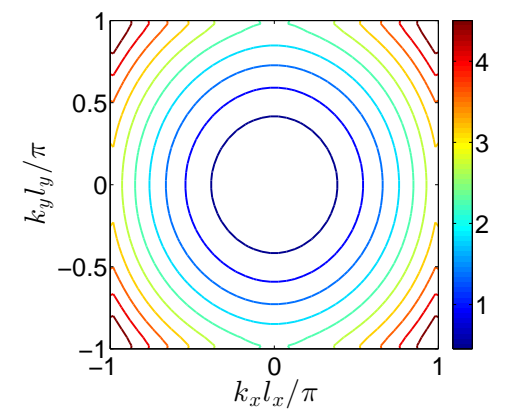

(a)

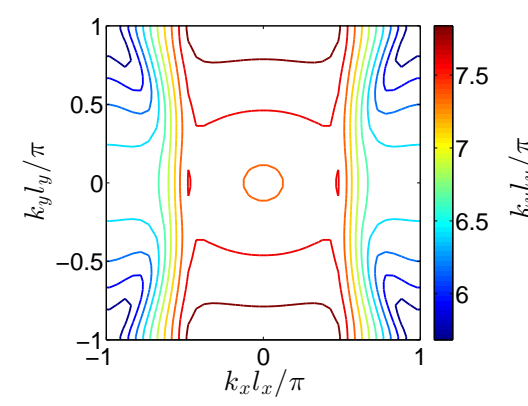

(c)

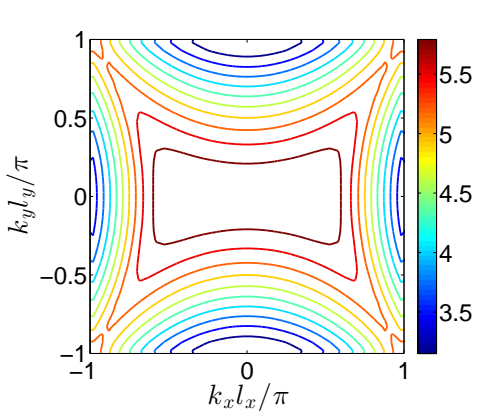

(b)

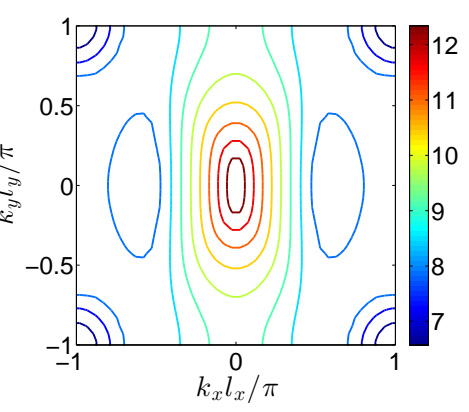

(d)

Figure 5: Contour plots of dispersion surfaces for the first wave modes (contour frequency values are expressed in $\mathrm{kHz}$ ). (a) Mode 1. (b) Mode 2. (c) Mode 3. (d) Mode 4.

Additional observations can be made upon the detailed analysis of the out-of-plane branches of the dispersion diagram, which are shown in Fig. 6. In this frequency range, the two largest partial bandgaps occur in the O-C range with center frequencies of approximately 6.75 and $20 \mathrm{kHz}$. These frequencies are highlighted by a dashed and solid line, respectively. The O-C direction corresponds to the vertical direction in the plate, which suggests that within these bands motion occurs predominantly along the horizontal 
direction, thus indicating the directional behavior of wave motion. Furthermore, the branches bounding these partial bandgaps end with a zero slope at $\mathrm{O}$, which identifies a null group velocity. In particular, the lower bandgaps appears to be bounded by branches that remain relatively flat through the entire $\mathrm{O}-\mathrm{C}$ wavenumber range, which characterizes wave motion remaining localized as a result of the occurrence of internal resonant behavior. This is illustrated by plotting the wave modes associated with these branches. The local wave modes are plotted both at $\mathrm{O}$, which corresponds to the classic eigenmodes of the unit cell, and at the point half way in between $\mathrm{O}$ and $\mathrm{C}$. These mode shapes are all shown in Fig. 7, where it can be seen that the mode changes slightly along the branch.

The localized nature of the modes associated with the lowest two branches appears evident from Figs. 7 (a) and (b), which correspond to the modes belonging to the branches starting at points a) and b) in Fig. 6 for frequencies equal to 6.1 and $7.4 \mathrm{kHz}$. Both modes are characterized by the resonance of the double-C inclusion. One is bending dominated and the other being of a torsion type. The modes associated with the second partial bandgap (Figs. 7 (c) and (d)) appear different in nature as the corresponding dynamic deformations are no longer localized to the inclusion, but occur over the entire unit cell. However, the out-of-phase motion of the internal inclusion with respect to the surrounding medium appears as a common feature of all the modes shown in Fig. 7.

\subsection{Group velocity}

The group velocity is a vectorial quantity that provides information regarding the speed and direction of wave propagation in the considered medium 


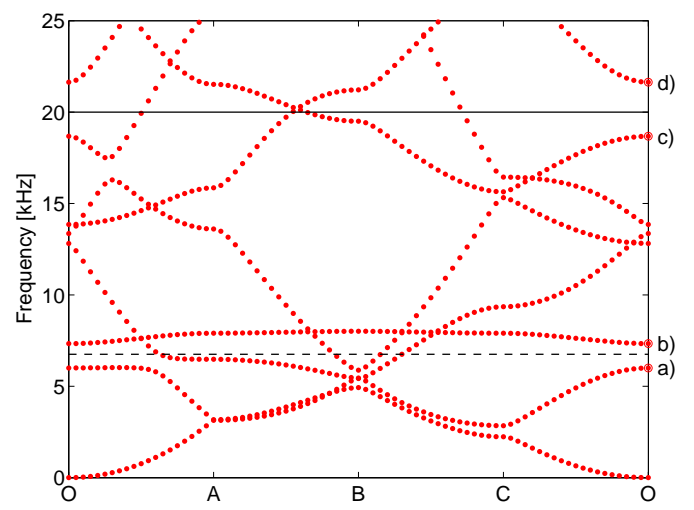

Figure 6: Illustration of partial bandgaps in the O-C range for out-of-plane polarized branches. Center frequencies of approximately 6.75 and $20 \mathrm{kHz}$ are, respectively, highlighted by the black dashed and solid lines.

[25]. The evaluation of group velocities relies on the estimation of the dispersion relations, which can be conducted using the procedure illustrated in the previous section. Subsequently, the estimation of the group velocity follows the approach originally presented in [32] for the case of $1 \mathrm{D}$ photonic crystal.

The group velocity $\mathbf{c}_{g}$, defined as

$$
\mathbf{c}_{g}=\nabla \omega=\left[\begin{array}{ll}
\frac{d \omega}{d k_{x}}, & \frac{d \omega}{d k_{y}}
\end{array}\right]^{T}
$$

is estimated by considering the form of the eigenvalue problem in terms of $\omega$ (Eq. (11)), i.e.:

$$
\mathbf{G}(\mathbf{k}, \omega(\mathbf{k}), \mathbf{u}(\mathbf{k}))=\left(\mathbf{K}-\omega^{2} \mathbf{M}\right) \mathbf{u}=\mathbf{0}
$$

Differentiating with respect to $\mathbf{k}$ gives:

$$
\frac{d \mathbf{G}(\mathbf{k}, \omega(\mathbf{k}), \mathbf{u}(\mathbf{k}))}{d \mathbf{k}}=\frac{\partial \mathbf{G}}{\partial \mathbf{u}} \frac{d \mathbf{u}}{d \mathbf{k}}+\frac{\partial \mathbf{G}}{\partial \omega} \frac{d \omega}{d \mathbf{k}}+\frac{\partial \mathbf{G}}{\partial \mathbf{k}}
$$




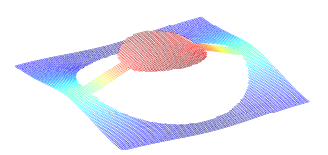

(a)

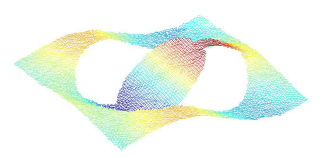

(c)
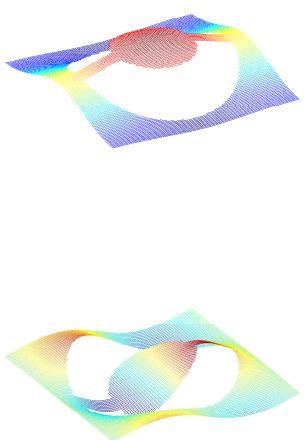

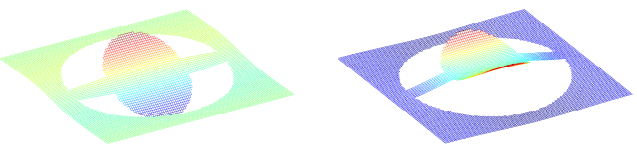

(b)

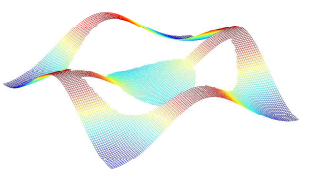

(d)

Figure 7: Wave mode shapes associated with the branches bounding the partial bandgaps. For each branch two modes have been plotted (left is at $\mathrm{O}$, right is halfway between $\mathrm{O}$ and $\mathrm{C}$ ). Modes belonging to branch starting at (a) $6.0 \mathrm{kHz}$. (b) $7.3 \mathrm{kHz}$. (c) $18.7 \mathrm{kHz}$. (d) $21.6 \mathrm{kHz}$.

The term $d \mathbf{u} / d \mathbf{k}$ can be eliminated by pre-multiplying by a vector $\mathbf{v}^{T}$ :

$$
\mathbf{v}^{T} \frac{\partial \mathbf{G}}{\partial \mathbf{u}} \frac{d \mathbf{u}}{d \mathbf{k}}+\mathbf{v}^{T} \frac{\partial \mathbf{G}}{\partial \omega} \frac{d \omega}{d \mathbf{k}}+\mathbf{v}^{T} \frac{\partial \mathbf{G}}{\partial \mathbf{k}}=0
$$

and requiring that:

$$
\mathbf{v}^{T} \frac{\partial \mathbf{G}}{\partial \mathbf{u}}=\mathbf{0}
$$

which corresponds to a left eigenvalue problem. Substituting Eq. (17) into Eq. (15), the group velocity can be obtained as:

$$
\frac{d \omega}{d \mathbf{k}}=-\frac{\mathbf{v}^{T} \frac{\partial \mathbf{G}}{\partial \mathbf{k}}}{\mathbf{v}^{T} \frac{\partial \mathbf{G}}{\partial \omega}}
$$


with

$$
\begin{aligned}
& \frac{\partial \mathbf{G}}{\partial k_{x}}=\left(-i \mathbf{K}_{1}+2 k_{x} \mathbf{K}_{3}+k_{y} \mathbf{K}_{4}\right) \mathbf{u} \\
& \frac{\partial \mathbf{G}}{\partial k_{y}}=\left(-i \mathbf{K}_{2}+k_{x} \mathbf{K}_{4}+2 k_{y} \mathbf{K}_{5}\right) \mathbf{u} \\
& \frac{\partial \mathbf{G}}{\partial \omega}=-2 \omega \mathbf{M} \mathbf{u}
\end{aligned}
$$

In general, it is necessary to solve the eigenvalue problem in Eq. 17 to get the left eigenvector $\mathbf{v}$. However, if the presence of any dissipation mechanism is neglected, both $\mathbf{K}$ and $\mathbf{M}$ are Hermitian and the left eigenvector $\mathbf{v}$ is the complex conjugate of the right eigenvector, $\mathbf{u}$.

In order to compute the group velocity at a given frequency $\omega$, we need to know the corresponding wave vector(s) k. Eq. (11) is a quadratic eigenvalue problem in both $k_{x}$ and $k_{y}$. However, by introducing an angle, $\theta$, specifying the direction of a given wave vector, the wave vector is given as:

$$
\mathbf{k}=\left[k_{x}, k_{y}\right]^{T}=[k \cos \theta, k \sin \theta]^{T}
$$

such that Eq. (11) can be expressed as a standard eigenvalue problem in terms of the wave vector magnitude $k$ [33]:

$$
\left(\mathbf{K}_{k}(\theta, \omega)-k \mathbf{M}_{k}(\theta)\right) \mathbf{u}_{k}=\mathbf{0}
$$

Equation (22) can be solved in terms of the wave vector for an assigned frequency $\omega$ and direction as defined by the angle $\theta$.

Examples of group velocity plots computed at two selected frequencies are shown in Figs. 8 (b) and (d), along with the corresponding isofrequency contours of the dispersion surfaces (Figs. 8 (a) and (c)). The isofrequency 
dispersion contour at $2 \mathrm{kHz}$ appears as almost circular, which is an indication of the quasi-isotropic behavior of the plate at low frequencies. The group velocity directional plot at the same frequency shows that propagation appears as occurring with a slightly higher velocity along the horizontal direction, as shown by the lobar shape of the group velocity curve (Fig. 8 (b)). The case of $11 \mathrm{kHz}$ includes two sets of curve, as two wave modes contribute to propagation at this frequency (Figs. 8 (c) and (d)). The group velocity variation in Fig. 8 (d) shows two curves, one of which appears again elongated along the horizontal direction, to indicate faster propagation along the horizontal direction. The second curve is characterized by similar speeds along the vertical direction and longitudinal one, however it appears to be characterized by two sharper longitudinal lobes. The group velocity curves in Fig. 8 (b) and $(\mathrm{d})$ define the pattern of wave motion within the domain, and essentially predict the shape of the propagating wavefront at the considered frequencies. 


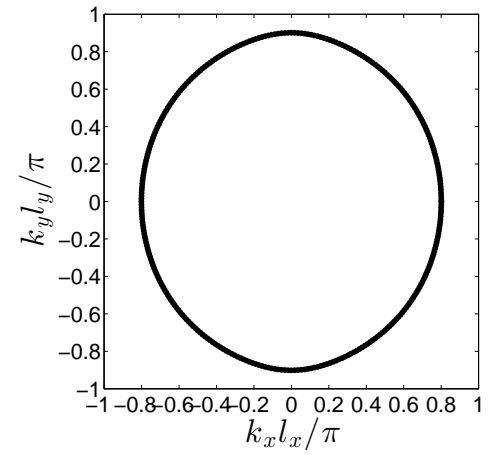

(a)

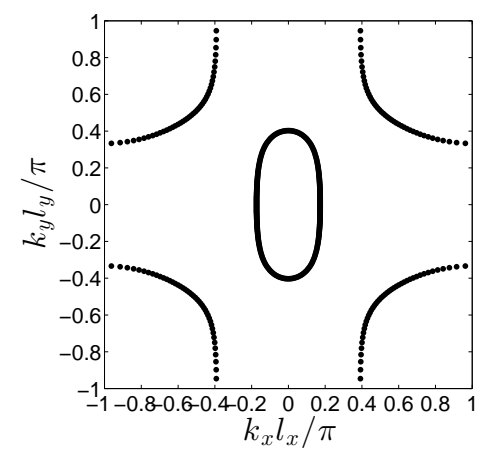

(c)

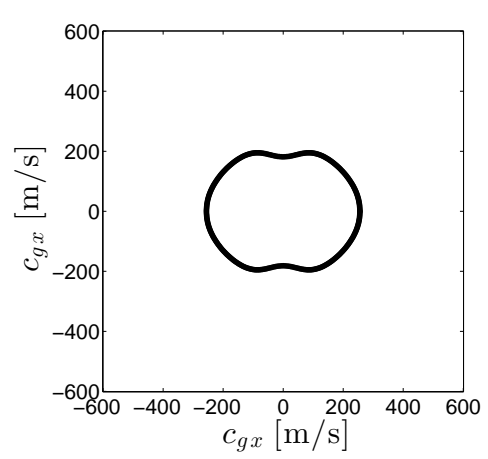

(b)

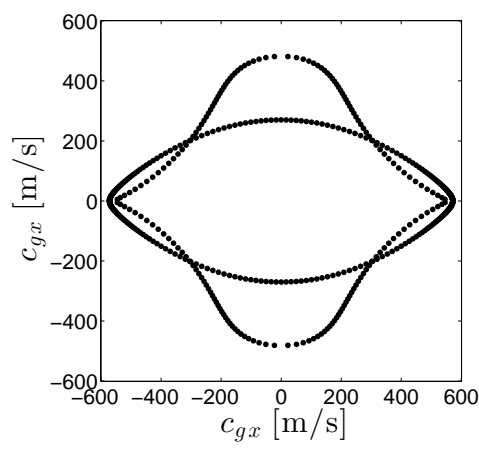

(d)

Figure 8: Isofrequency contour and corresponding group velocity profiles at two selected frequencies. (a) Isofrequency contour $2 \mathrm{kHz}$. (b) Group velocity $2 \mathrm{kHz}$. (c) Isofrequency contour $11 \mathrm{kHz}$. (d) Group velocity $11 \mathrm{kHz}$. 


\section{Experimental investigation of wave motion}

\subsection{Setup}

The wave propagation characteristics of the considered plate are investigated experimentally through measurements conducted using a scanning laser vibrometer (SLV) (Polytec Model PSV 400 M2). The plate specimen was fabricated out of an aluminum (Young's Modulus $E=69$ GPa, Poisson's ratio $\nu=0.33$, and the density $\rho=2700 \mathrm{~kg} / \mathrm{m}^{3}$ ) plate of the dimensions considered for the numerical investigations. A $15 \times 15$ array of double-C perforations was obtained through water jet cutting. The resulting plate is shown in Fig. 9, along with the detail of a unit cell. The center cell was not cut in order to maintain a central location where a piezoelectric (PZT) disc is bonded to apply the desired excitation. A PZT disc of $7 \mathrm{~mm}$ in diameter from Steminc Corp. was selected as a source capable of introducing the desired excitations at the frequencies/wavelengths of interest to the current study. The choice is based on prior experience on guided wave fields generation and detection in plate structures as described for example in [34]. The piezoelectric disc is excited by a seven cycle Hanning modulated sine burst at the desired center frequency. The plate's out-of-plane response is recorded over a refined grid of points prescribed through the SLV software. At each measurement point, the recorded response is the result of ten averages taken to minimize distortions due to measurement noise. Complete wave field images are obtained by aggregating single-point measurements at each grid point. Measurements and excitations are synchronized with a trigger signal so that proper phase is maintained among all measurement points. Upon completing the scanning of the measurement grid, the data are post-processed by the SLV software and 


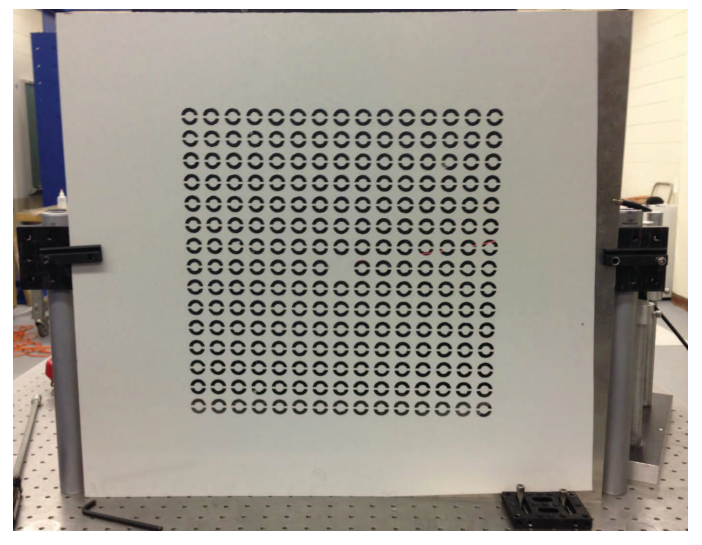

(a)

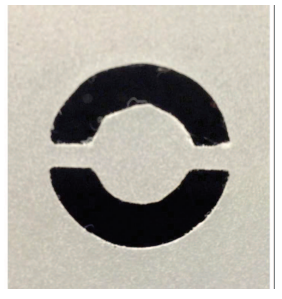

(b)

Figure 9: (a) Experimental plate and (b) close-up containing detail of unit cell.

coherent wave images are obtained. Examples of wave field images obtained for two of the excitation frequencies considered are shown in Figs. 10 and 11.

\subsection{Wave modes}

A set of verification experiments is conducted to evaluate the modal characteristics of the unit cell at some of the frequencies highlighted by the numerical study. These experiments are conducted by considering a refined local grid over a single unit cell. The plate is excited through harmonic excitation at the frequencies of interest. Frequency domain acquisitions are conducted to measure the dynamic deflection shapes of the unit cell, which are regarded as closely related to the unit cell wave modes of the plate. Results at three selected frequencies displayed in Fig. 11 show significant resemblance to the wave modes presented in Fig. 7. This once again confirms the accuracy of 


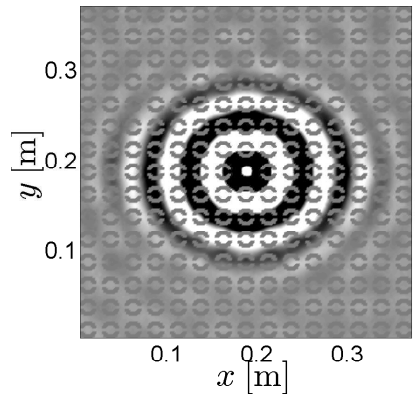

(a)

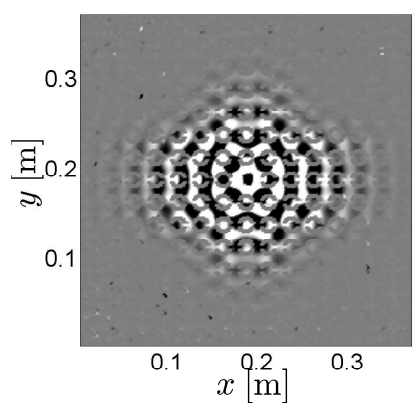

(d)

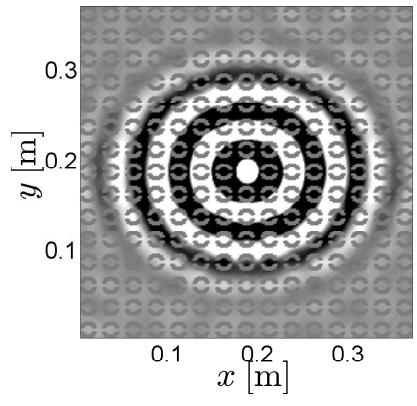

(b)

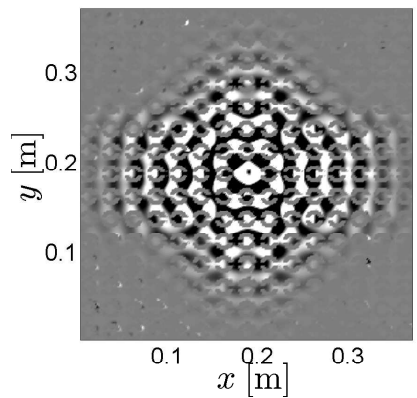

(e)

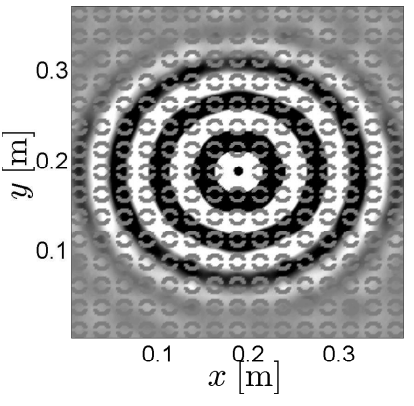

(c)

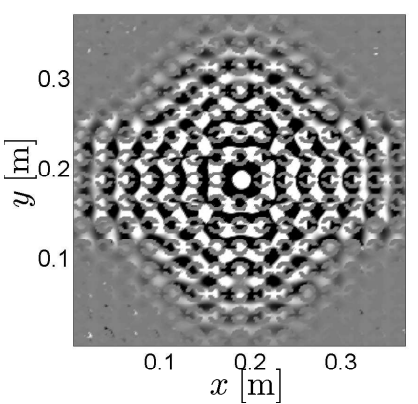

(f)

Figure 10: Snapshots of experimental wave fields for excitation at $3 \mathrm{kHz}$ after (a) $t=546 \mu \mathrm{s}$, (b) $t=624 \mu \mathrm{s}$, and (c) $t=702 \mu \mathrm{s}$, and for excitation at $11 \mathrm{kHz}$ after (d) $t=312 \mu \mathrm{s}$, (e) $t=390 \mu \mathrm{s}$, and (f) $t=468 \mu \mathrm{s}$.

the numerical implementation presented in the first part of this paper, and its ability to predict local resonance characteristics at the unit cell level.

\subsection{Evaluation of dispersion}

The dispersion relations for the plate are estimated by processing the wave field data recorded during the experiments. Wave field information can be described as an array of matrices $w(x, y, t)$ containing spatial and temporal field information, where $w$ denotes the measured out-of-plane displacement 


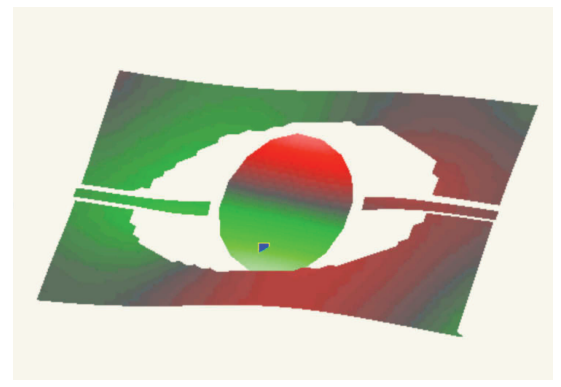

(a)

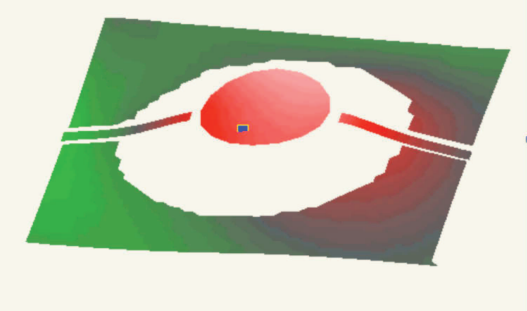

(b)

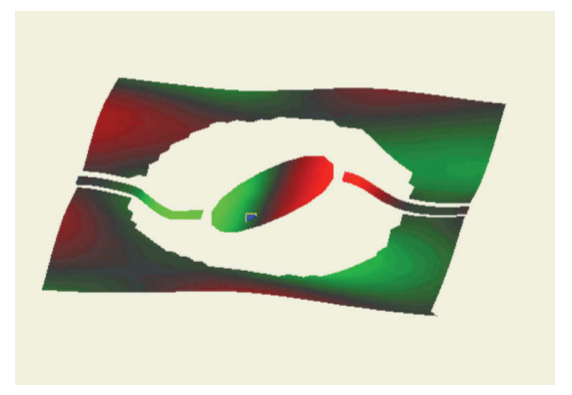

(c)

Figure 11: Dynamic deformed shapes at selected frequencies recorded through local measurements at the unit cell level. (a) $6.0 \mathrm{kHz}$. (b) 7.3 kHz. (c) $19.3 \mathrm{kHz}$.

of the plate.

The dispersion properties are first estimated along the O-A and $\mathrm{O}-\mathrm{C}$ directions - in order to describe the relation between frequency and wavenumber along two of the boundaries of the Brillouin zone - corresponding to the horizontal and vertical directions, respectively. To this end, one of the coordinates is kept fixed in the wave field matrix, and the two-dimensional Fourier transform (2D-FT) in time and space (along the remaining coordinate and time) is evaluated. This operation can be described as follows: 


$$
\begin{aligned}
\hat{w}\left(k_{x}, \omega\right) & =\mathcal{F}_{2 D}\left[w\left(x, y=y_{0}, t\right)\right] \\
\hat{w}\left(k_{y}, \omega\right) & =\mathcal{F}_{2 D}\left[w\left(x=x_{0}, y, t\right)\right]
\end{aligned}
$$

where $\mathcal{F}_{2 D}$ denotes the $2 \mathrm{D}-\mathrm{FT}$ operator, $\hat{w}$ is the representation of the plate response in the frequency/wavenumber $(f-k)$ domain, while $x_{0}, y_{0}$ are the coordinates along which dispersion is estimated.

The process is conducted by extracting data from the measurement grid shown in Fig. 12, specifically along the horizontal and vertical line shown in the figure, which correspond to the O-A and O-C directions of the band diagram. The procedure is applied to data corresponding to various excitation frequencies, such that a broader band of the spectrum can be covered by superimposing the results of several narrowband experiments. Results for the $\mathrm{O}-\mathrm{A}$ and $\mathrm{O}-\mathrm{C}$ directions are presented in Fig. 13, where the experimental contours (from five experiments with excitation frequencies $2 \mathrm{kHz}, 3$ $\mathrm{kHz}, 8.5 \mathrm{kHz}, 11 \mathrm{kHz}$, and $15 \mathrm{kHz}$ ) are overlaid to the numerically estimated branches for the corresponding directions. As one can easily see, the experimental branches appear in a wavenumber range that exceeds the limits of the first Brillouin zone, as the spatial sampling considered includes several measurement points within each unit cell. In order to enable a comparison with numerical results, the numerical branches are therefore replicated periodically over subsequent intervals in multiples of $\pi$ in order to observe or resolve potential indetermination associated with branch folding, which is the result of the investigation of Bloch wave modes.

Next, dispersion relations are visualized in the $k_{x}, k_{y}$ domain through the application of the 3D FT and the selection of specific frequencies of interest. 


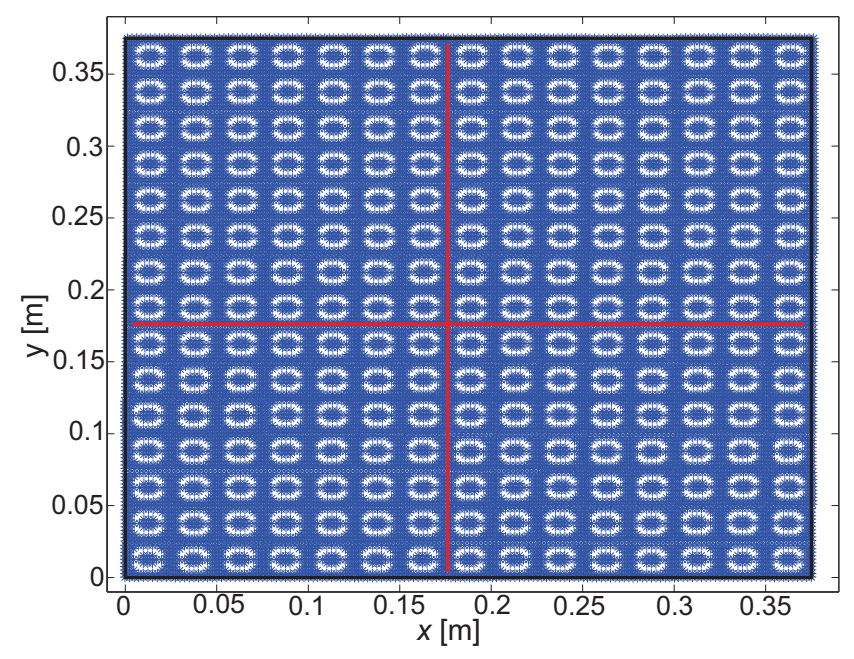

Figure 12: Experimental grid. Red lines indicate which measurement points are used for evaluation of dispersion relation for O-A (horizontal line) and O-C (vertical line).

This operation can be described as follows:

$$
\hat{w}\left(k_{x}, k_{y}, \omega\right)=\mathcal{F}_{3 D}[w(x, y, t)]
$$

where $\mathcal{F}_{3 D}$ denotes the $3 \mathrm{D}$ Fourier transform. Representation of $\hat{w}$ at a specified frequency $\omega=\omega_{0}$ leads to a surface $\hat{w}\left(k_{x}, k_{y}, \omega_{0}\right)$ whose magnitude can be represented in the $k_{x}, k_{y}$ domain, where direct comparison with the dispersion surfaces at the selected frequency can be conducted.

The dispersion surfaces are evaluated through the Bloch analysis, as presented in the previous sections, conducted over a specified grid of the in-plane wavenumber components. The isofrequency line of interest at $\omega=\omega_{0}$ is extracted and overlaid to the experimental contour, as shown in Fig. 14. This representation allows the evaluation of the energy content in the wavenum- 
ber domain, and the evaluation of potential directionality induced by the anisotropy of the plate at the selected frequencies. In Fig. 14, the numerical contours are black lines (Eq. 11) and dots (Eq. 22), while the surfaces $\left|\hat{w}\left(k_{x}, k_{y}, \omega_{0}\right)\right|$, each normalized to unity for convenience of representation, is shown by the different contours, with red representing the highest values and blue the lower values. For clarity, the boundaries of the first Brillouin zone are also highlighted by the blue square included in the figures. As expected, the numerical solutions found using Eq. 11 coincide with the solutions to Eq. 22. The experimental results confirm the previously observed fact that the measured wavenumbers exceed the boundaries of the Brillouin zone, which requires for the numerical dispersion to be computed beyond this range.

The numerical results show the expected periodicity in the wavenumber domain, and generally overlay well with the experimental results. At low frequencies, the results indicate that the plate behavior is approximately isotropic, as demonstrated by the quasi-circular isofrequency lines obtained experimentally and numerically (see Figs. 14 (a) and (b) for results corresponding to $2 \mathrm{kHz}$ and $3 \mathrm{kHz}$, respectively). At higher frequencies (see Figs. 14 (c) and (d) for results corresponding to $8.5 \mathrm{kHz}$ and $11 \mathrm{kHz}$, respectively) the distributions show occurrences of preferential directions corresponding to peaks of the surface $\left|\hat{w}\left(k_{x}, k_{y}, \omega_{0}\right)\right|$ in the wavenumber domain. A good agreement is observed, in particular for the low frequency cases. At higher frequencies, the predictions appear less accurate which may be attributed to the reduced fidelity of the model and to the contribution of other wave modes not included in the numerical isofrequency contour displayed. 


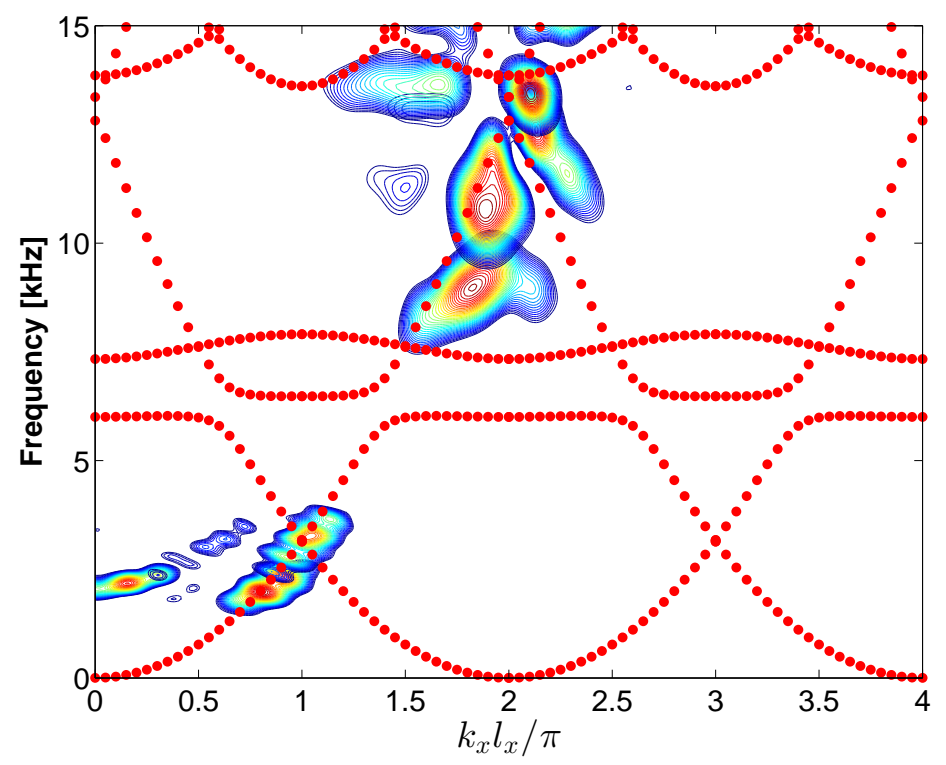

(a)

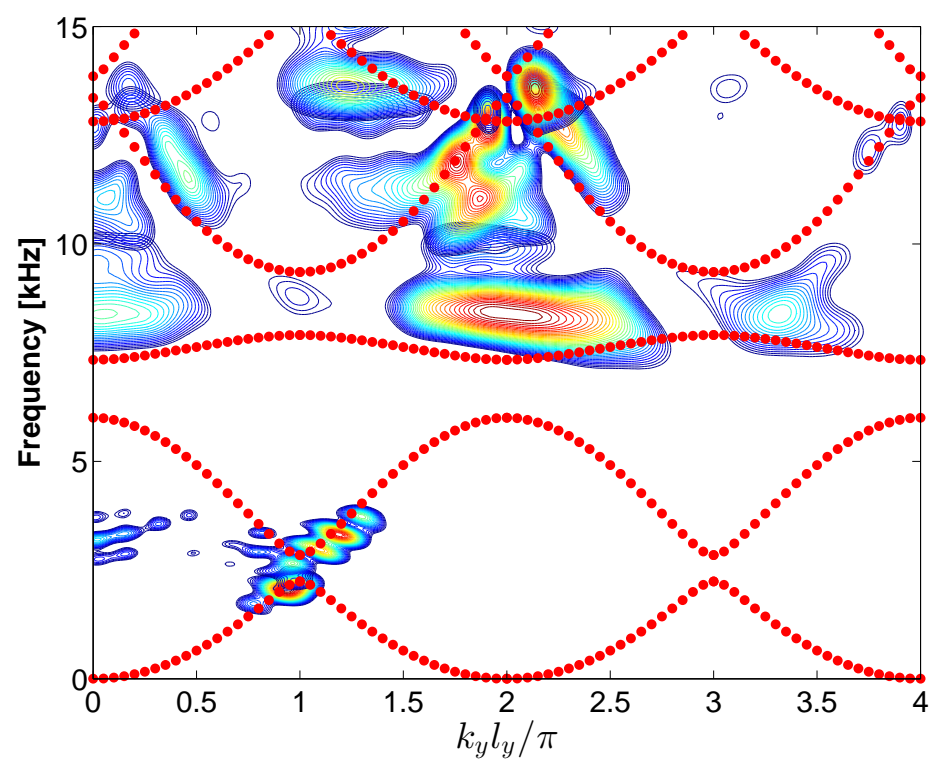

(b)

Figure 13: Dispersion relations $k-f$ representation along the (a) O-A and (b) O-C direction, and comparison with numerical predictions (red dotted lines) (Numerical branches are replicated periodically in intervals of $\pi$ ). 


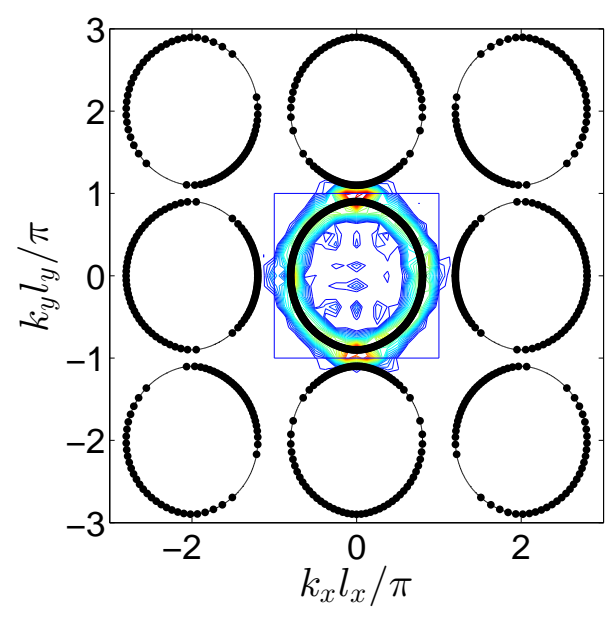

(a)

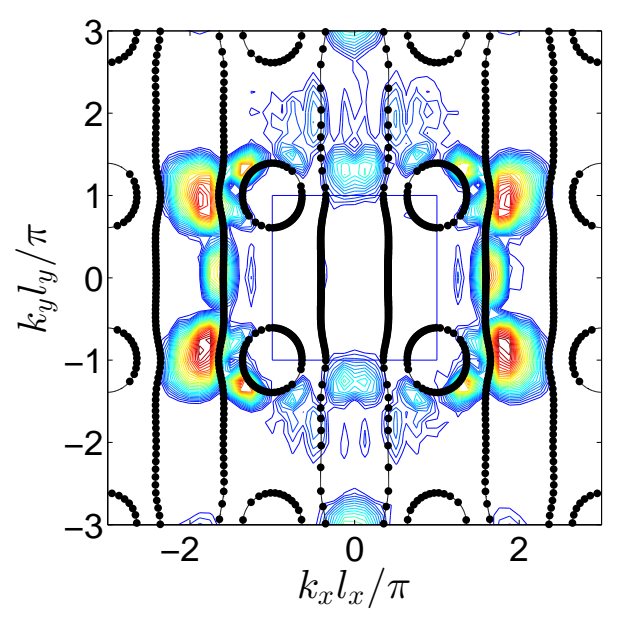

(c)

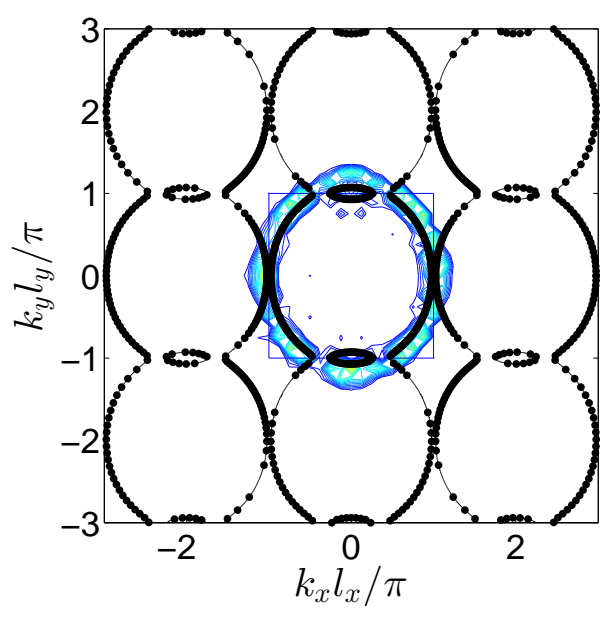

(b)

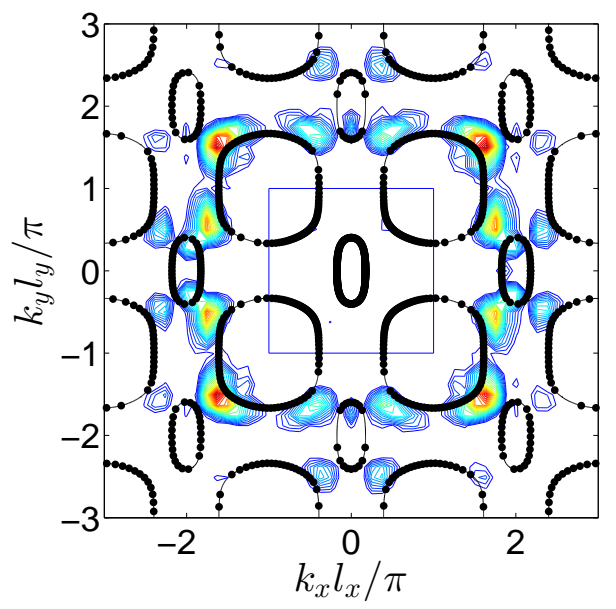

(d)

Figure 14: Experimental 3D FT (contour plots) and isofrequency dispersion surfaces (black dotted line) at selected frequencies for representation of plate dispersion in the $k_{x}-k_{y}$ domain. (a) $2 \mathrm{kHz}$. (b) $3 \mathrm{kHz}$. (c) $8.5 \mathrm{kHz}$. (d) $11 \mathrm{kHz}$. Note that the numerical results are plotted both as contour slices (solid black line), and as solutions to Eq. 22 (black dots) in which the angle and frequency are specified before solving for $k$. 


\subsection{Directionality and group velocities}

The dispersion plots presented in Fig. 14 reveal the complex plate behavior as the frequency increases, which is characterized by a transition from quasi-isotropic motion, to a more complex pattern with some distinct preferential directions. This can be further investigated from the analysis of group velocity at the selected frequencies and their representation in conjunction with time snapshots of the wave field.

In theory, the directional group velocity plots of the kind shown in Fig. 8 describe the pattern of energy flow within the structure, and highlight the concentration of wave vectors in preferential orientations. This is expected to be reflected in the shape of the wavefront observed at specific time instants. This representation is provided in Fig. 15, where the group velocity is overlaid on the wave field generated by excitation at the four selected frequency values. The group velocity and wave field snapshots confirm the quasi-isotropic behaviors at $2 \mathrm{kHz}$, and show distinct directional characteristics at the higher frequencies where wave motion occurs preferentially along the horizontal direction at $8.5 \mathrm{kHz}$ (see Fig. 15 (c)) and both the horizontal and vertical direction as in the case of the wave field at $11 \mathrm{kHz}$ shown in Fig. $15(\mathrm{~d})$.

In all cases presented, the group velocity predictions provide a good representation of the wavefront and agree with the experimental measurements. Such agreement is somewhat confounded by the fact the excitation does not consist of a pure tone, but it is defined by a seven cycle burst, which therefore includes a range of frequencies that partially excites other wave modes. 


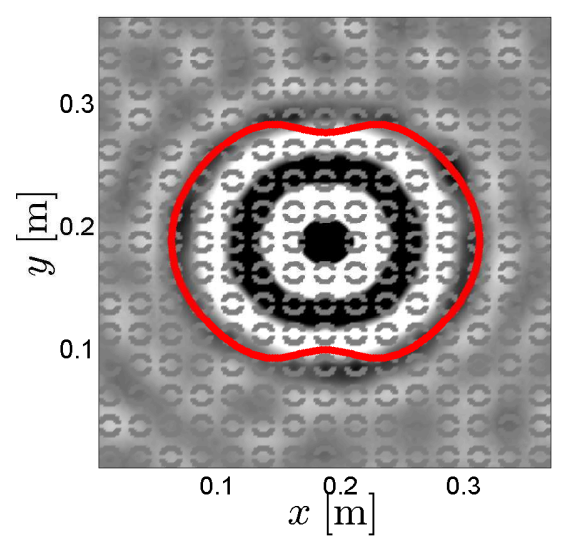

(a)

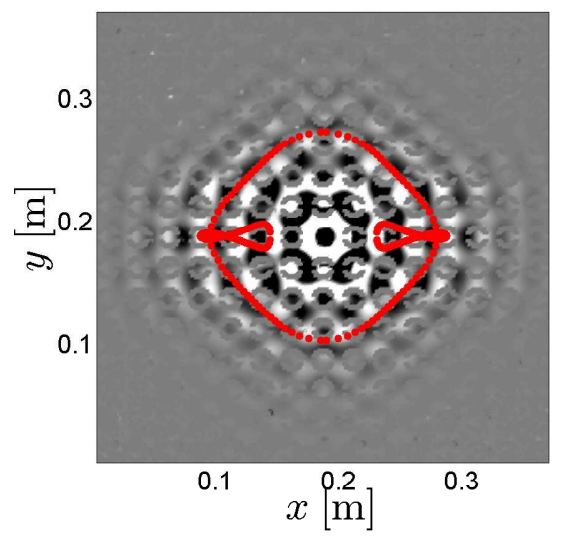

(c)

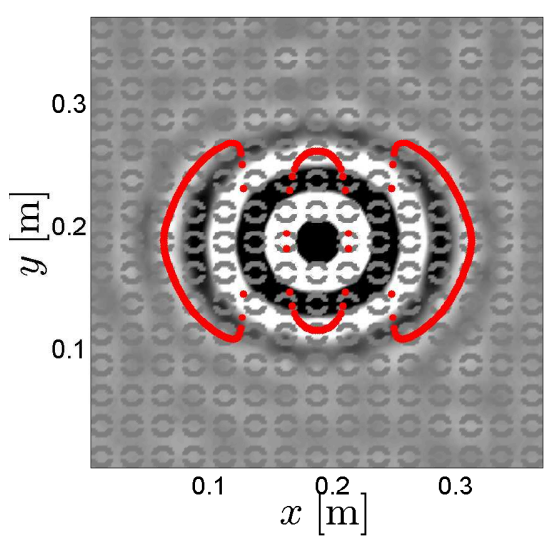

(b)

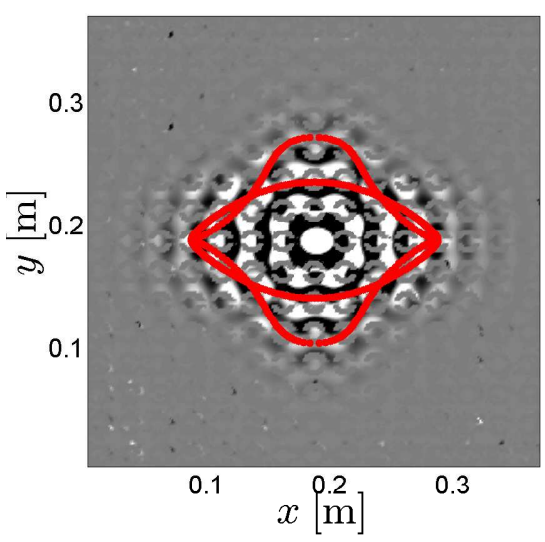

(d)

Figure 15: Experimental wave field and theoretical group velocities at the corresponding excitation frequencies. (a) $2 \mathrm{kHz}$. (b) $3 \mathrm{kHz}$. (c) $8.5 \mathrm{kHz}$. (d) $11 \mathrm{kHz}$. 
Further, quantitative insight into the directional behavior of the plate can be gained by evaluating the envelope of a propagating waveform. Envelope estimation is conducted through the application of the Hilbert transform (HT) along a selected direction of propagation. The envelope evolution along one of the coordinates, say the horizontal direction $x$, is estimated as:

$$
\mathcal{E}(x, t)=\left|\mathcal{H}\left[w\left(x, y=y_{0}, t\right)\right]\right|
$$

where $\mathcal{H}$ denotes the $\mathrm{HT}$ of the considered waveform.

The envelopes' evolution at two frequency values along the $x$ - and $y$ directions are shown in Fig. 16 in the form of space-time amplitude maps on a gray scale (with the black color being associated with the highest amplitude values). The plots also show propagation lines along the considered directions obtained by considering the numerically computed group velocity component along the considered directions. The lines, represented as red dotted lines, are obtained from expressions of the kind of $\pm c_{g_{x}} t$ and $\pm c_{g_{y}} t$. These representations again confirm the accurate estimations of group velocities obtained from the considered numerical model, which provides the correct rate of envelope propagation. The plots in Fig. 16 clearly highlight the different wave speeds for the considered mode of propagation along the two principal directions of motion. 


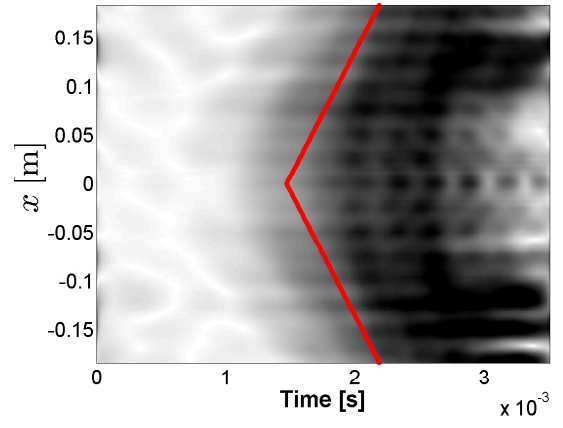

(a)

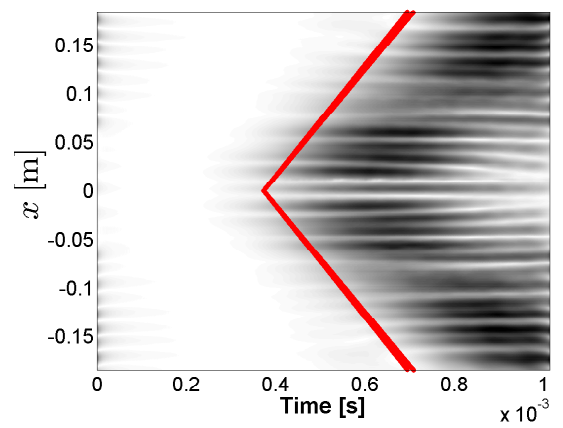

(c)

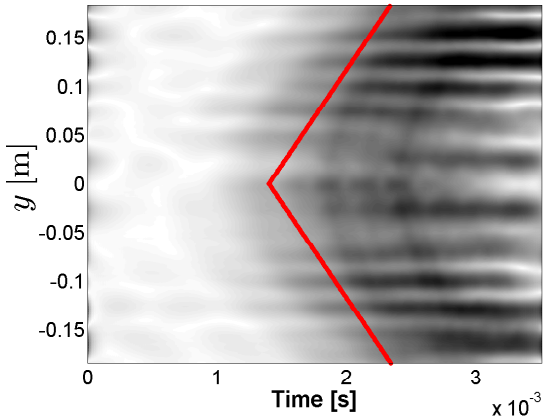

(b)

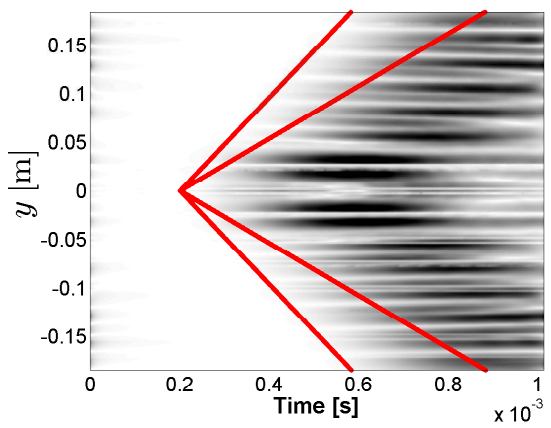

(d)

Figure 16: Space time evolution of wave motion along the vertical and horizontal direction and rate of propagation predicted numerically through the estimation of group velocity (red solid line). (a) $2 \mathrm{kHz}, x$-propagation. (b) 2 kHz, $y$-propagation. (c) $11 \mathrm{kHz}, x$-propagation. (d) $11 \mathrm{kHz}, y$-propagation. 


\section{Conclusion}

The work presented the numerical and experimental investigation of wave motion in a periodic plate with double-C perforations. The considered configuration was chosen because of its expected non-isotropic equivalent behavior, which leads to directional wave motion. Furthermore, the periodic arrangement of perforations produces partial bandgaps due to internal resonances. These wave phenomena are first predicted through a numerical model formulated using MP elements, and are then investigated experimentally through the analysis of wave field images. Numerical and experimental results characterize the 2D dispersion properties of the plate, and its group velocities as a function of direction of propagation and frequency. Furthermore, detailed modeling and measurements at the unit cell level identify modes of resonance associated with partial bandgaps. The results presented in this study illustrate the range of investigations that can be performed on periodic plate waveguides and the wave properties that can be estimated through the presented numerical and experimental approaches. In addition, the considered configuration can be used as a baseline for the design of planar waveguides with desired directional and bandgap configurations through unit cell topology optimization.

\section{Acknowledgement}

E. Andreassen's support for this work was provided by the Danish Research Agency through the innovation consortium F•MAT. M. Ruzzene and K. Manktelow acknowledge the support from the National Science Foundation under grants no. 0926776 and 1130368. 


\section{References}

[1] G. M. Burrow, T. K. Gaylord, Multi-beam interference advances and applications: Nano-electronics, photonic crystals, metamaterials, subwavelength structures, optical trapping, and biomedical structures, Micromachines 2 (2011) 221-257.

[2] M. L. Povinelli, S. G. Johnson, J. D. Joannopoulos, J. B. Pendry, Toward photonic-crystal metamaterials: Creating magnetic emitters in photonic crystals, Applied Physics Letters 82 (2003).

[3] E. Yablonovitch, T. Gmitter, Photonic band structure: The facecentered-cubic case, Phys. Rev. Lett. 63 (1989) 1950-1953.

[4] M. Maldovan, Sound and heat revolutions in phononics, Nature 503 (2013) 209-217.

[5] S. Guenneau, A. Movchan, G. Pétursson, S. A. Ramakrishna, Acoustic metamaterials for sound focusing and confinement, New Journal of Physics 9 (2007) 399.

[6] M. Farhat, S. Guenneau, S. Enoch, A. B. Movchan, G. G. Petursson, Focussing bending waves via negative refraction in perforated thin plates, Applied Physics Letters 96 (2010) 081909.

[7] M. Farhat, S. Guenneau, S. Enoch, High directivity and confinement of flexural waves through ultra-refraction in thin perforated plates, EPL (Europhysics Letters) 91 (2010) 54003. 
[8] J. Pierre, O. Boyko, L. Belliard, J. O. Vasseur, B. Bonello, Negative refraction of zero order flexural lamb waves through a two-dimensional phononic crystal, Applied Physics Letters 97 (2010) 121919.

[9] M. Ruzzene, F. Scarpa, F. Soranna, Wave beaming effects in twodimensional cellular structures, Smart Materials and Structures 12 (2003) 363.

[10] F. R. Montero de Espinosa, E. Jiménez, M. Torres, Ultrasonic band gap in a periodic two-dimensional composite, Phys. Rev. Lett. 80 (1998) $1208-1211$.

[11] O. Sigmund, J. S. Jensen, Systematic design of phononic band-gap materials and structures by topology optimization, Philos. Trans. R. Soc. Lond. A, Math. Phys. Eng. Sci. (UK) Philosophical transactions - Royal Society. Mathematical, Physical and engineering sciences 361 (2003) 1001-1019.

[12] S. Halkjaer, O. Sigmund, J. S. Jensen, Inverse design of phononic crystals by topology optimization, Zeitschrift für Kristallographie - Crystalline Materials 220 (2005) 895-905.

[13] O. R. Bilal, M. I. Hussein, Ultrawide phononic band gap for combined inplane and out-of-plane waves., Physical Review E: Statistical, Nonlinear \& Soft Matter Physics 84 (2011)-.

[14] T.-T. Wu, J.-C. Hsu, J.-H. Sun, Phononic plate waves, Ultrasonics, Ferroelectrics and Frequency Control, IEEE Transactions on 58 (2011) $2146-2161$. 
[15] Y. Pennec, B. Djafari-Rouhani, H. Larabi, J. O. Vasseur, A. C. HladkyHennion, Low-frequency gaps in a phononic crystal constituted of cylindrical dots deposited on a thin homogeneous plate, Physical Review B 78 (2008) 104105.

[16] A. Khelif, B. Djafari-Rouhani, J. O. Vasseur, P. A. Deymier, P. Lambin, L. Dobrzynski, Transmittivity through straight and stublike waveguides in a two-dimensional phononic crystal, Phys. Rev. B 65 (2002) 174308174308.

[17] M. Oudich, M. Senesi, M. B. Assouar, M. Ruzzene, J. H. Sun, B. Vincent, Z. L. Hou, T. T. Wu, Experimental evidence of locally resonant sonic band gap in two-dimensional phononic stubbed plates, Physical Review B 84 (2011).

[18] M. Badreddine Assouar, M. Senesi, M. Oudich, M. Ruzzene, Z. Hou, Broadband plate-type acoustic metamaterial for low-frequency sound attenuation, Applied Physics Letters 101 (2012) 173505-173505.

[19] K. F. Graff, Wave Motion in Elastic Solids, 1975.

[20] R. D. Cook, D. S. Malkus, M. E. Plesha, R. J. Witt, Concepts and Applications of Finite Element Analysis, fourth ed., John Wiley and Sons, 2002.

[21] R. Shelby, D. Smith, S. Nemat-Nasser, S. Schultz, Microwave transmission through a two-dimensional, isotropic, left-handed metamaterial, Applied Physics Letters 78 (2001) 489-491. 
[22] Z. Liu, X. Zhang, Y. Mao, Y. Y. Zhu, Z. Yang, C. T. Chan, P. Sheng, Locally resonant sonic materials, Science 289 (2000) 1734.

[23] S. Gonella, A. Spadoni, M. Ruzzene, F. Scarpa, Wave propagation and band-gap characteristics of chiral lattices, 21st Biennial Conference on Mechanical Vibration and Noise, Las Vegas (2007) 505-515.

[24] T. E. Michaels, J. E. Michaels, M. Ruzzene, Frequency-wavenumber domain analysis of guided wavefields, Ultrasonics 51 (2011) 452-466.

[25] L. Brillouin, Wave propagation in periodic structures, 2 ed., Dover Publications, 1953.

[26] R. S. Langley, The response of two-dimensional periodic structures to point harmonic forcing, Journal of Sound and Vibration 197 (1996) 469.

[27] J. Wolfe, Imaging phonons: acoustic wave propagation in solids, Cambridge Univ Pr, 1998.

[28] H. H. Huang, C. T. Sun, Wave attenuation mechanism in an acoustic metamaterial with negative effective mass density, New Journal of Physics 11 (2009) 013003.

[29] N. Fang, D. Xi, J. Xu, M. Ambati, W. Srituravanich, C. Sun, X. Zhang, Ultrasonic metamaterials with negative modulus, Nature Materials 5 (2006) 456.

[30] O. C. Zienkiewicz, R. L. Taylor, The Finite Element Method. Fifth Edition. Volume 2: Solid Mechanics, Butterworth-Heinemann, 2000. 
[31] E. Andreassen, J. S. Jensen, Analysis of phononic bandgap structures with dissipation, Journal of Vibration and Acoustics 135 (2013) 0410158.

[32] F. Wang, J. S. Jensen, O. Sigmund, J. Mørk, Systematic design of loss-engineered slow-light waveguides, Journal of the Optical Society of America A: Optics and Image Science, and Vision 29 (2012) 2657-2666.

[33] R. P. Moiseyenko, V. Laude, Material loss influence on the complex band structure and group velocity in phononic crystals, Physical Review B 83 (2011) 064301.

[34] M. Romanoni, S. Gonella, N. Apetre, M. Ruzzene, Two-dimensional periodic actuators for frequency-based beam steering, Smart Materials and Structures 18 (2009) 125023. 


\section{Appendix A. System matrices}

With the nodal displacements interpolated over the element using the shape function matrices $\mathbf{N}_{w}$ and $\mathbf{N}_{\Theta}$, as follows

$$
\mathbf{u}^{e}=\left[\begin{array}{cc}
\mathbf{N}_{w}(x, y) & \mathbf{0} \\
\mathbf{0} & \mathbf{N}_{\Theta}(x, y)
\end{array}\right]\left[\begin{array}{c}
\tilde{\mathbf{w}}^{e} \\
\tilde{\boldsymbol{\Theta}}^{e}
\end{array}\right]
$$

the corresponding expressions for the element matrices, in Eq. 11 are:

$$
\begin{aligned}
& \mathbf{k}_{0}^{e}=\int_{\Omega^{e}}\left[\begin{array}{cc}
-\nabla \mathbf{N}_{w}^{T} \mathbf{G} \nabla \mathbf{N}_{w} & \left(\mathbf{N}_{\Theta}^{T} \mathbf{G} \nabla \mathbf{N}_{w}\right)^{T} \\
\mathbf{N}_{\Theta}^{T} \mathbf{G} \boldsymbol{\nabla} \mathbf{N}_{w} & -\boldsymbol{\partial} \mathbf{N}_{\Theta}^{T} \mathbf{D} \boldsymbol{\partial} \mathbf{N}_{\Theta}-\mathbf{N}_{\Theta}^{T} \mathbf{G} \mathbf{N}_{\Theta}
\end{array}\right] d \Omega^{e} \\
& \mathbf{k}_{1}^{e}=-\int_{\Omega^{e}}\left[\begin{array}{cc}
\mathbf{N}_{w}^{T} \boldsymbol{\beta}_{1}^{T} \mathbf{G} \boldsymbol{\nabla} \mathbf{N}_{w}-\boldsymbol{\nabla} \mathbf{N}_{w}^{T} \mathbf{G} \boldsymbol{\beta}_{1} \mathbf{N}_{w} & -\left(\mathbf{N}_{\Theta}^{T} \mathbf{G} \boldsymbol{\beta}_{1} \mathbf{N}_{w}\right)^{T} \\
\mathbf{N}_{\Theta}^{T} \mathbf{G} \boldsymbol{\beta}_{1} \mathbf{N}_{w} & \mathbf{N}_{\Theta}^{T} \boldsymbol{\alpha}_{1}^{T} \mathbf{D} \partial \mathbf{N}_{\Theta}-\boldsymbol{\partial} \mathbf{N}_{\Theta}^{T} \boldsymbol{D} \boldsymbol{\alpha}_{1} \mathbf{N}_{\Theta}
\end{array}\right] d \Omega^{e} \\
& \mathbf{k}_{2}^{e}=-\int_{\Omega^{e}}\left[\begin{array}{cc}
\mathbf{N}_{w}^{T} \boldsymbol{\beta}_{2}^{T} \mathbf{G} \boldsymbol{\nabla} \mathbf{N}_{w}-\boldsymbol{\nabla} \mathbf{N}_{w}^{T} \mathbf{G} \boldsymbol{\beta}_{2} \mathbf{N}_{w} & -\left(\mathbf{N}_{\Theta}^{T} \mathbf{G} \boldsymbol{\beta}_{2} \mathbf{N}_{w}\right)^{T} \\
\mathbf{N}_{\Theta}^{T} \mathbf{G} \boldsymbol{\beta}_{2} \mathbf{N}_{w} & \mathbf{N}_{\Theta}^{T} \boldsymbol{\alpha}_{2}^{T} \mathbf{D} \boldsymbol{\partial} \mathbf{N}_{\Theta}-\boldsymbol{\partial} \mathbf{N}_{\Theta}^{T} \boldsymbol{D} \boldsymbol{\alpha}_{2} \mathbf{N}_{\Theta}
\end{array}\right] d \Omega^{e} \\
& \mathbf{k}_{3}^{e}=-\int_{\Omega^{e}}\left[\begin{array}{cc}
\mathbf{N}_{w}^{T} \boldsymbol{\beta}_{1}^{T} \mathbf{G} \boldsymbol{\beta}_{1} \mathbf{N}_{w} & \mathbf{0} \\
\mathbf{0} & \mathbf{N}_{\Theta}^{T} \boldsymbol{\alpha}_{1}^{T} \boldsymbol{D} \boldsymbol{\alpha}_{1} \mathbf{N}_{\Theta}
\end{array}\right] d \Omega^{e} \\
& \mathbf{k}_{4}^{e}=-\int_{\Omega^{e}}\left[\begin{array}{cc}
\mathbf{N}_{w}^{T}\left(\boldsymbol{\beta}_{1}+\boldsymbol{\beta}_{2}\right)^{T} \mathbf{G}\left(\boldsymbol{\beta}_{1}+\boldsymbol{\beta}_{2}\right) \mathbf{N}_{w} & \mathbf{0} \\
\mathbf{0} & \mathbf{N}_{\Theta}^{T}\left(\boldsymbol{\alpha}_{1}+\boldsymbol{\alpha}_{2}\right)^{T} \mathbf{D}\left(\boldsymbol{\alpha}_{1}+\boldsymbol{\alpha}_{2}\right) \mathbf{N}_{\Theta}
\end{array}\right] d \Omega^{e} \\
& \mathbf{k}_{5}^{e}=-\int_{\Omega^{e}}\left[\begin{array}{cc}
\mathbf{N}_{w}^{T} \boldsymbol{\beta}_{2}^{T} \mathbf{G} \boldsymbol{\beta}_{2} \mathbf{N}_{w} & \mathbf{0} \\
\mathbf{0} & \mathbf{N}_{\Theta}^{T} \boldsymbol{\alpha}_{2}^{T} \boldsymbol{D} \boldsymbol{\alpha}_{2} \mathbf{N}_{\Theta}
\end{array}\right] d \Omega^{e} \\
& \mathbf{m}^{e}=-\int_{\Omega^{e}}\left[\begin{array}{cc}
\rho h \mathbf{N}_{w}^{T} \mathbf{N}_{w} & \mathbf{0} \\
\mathbf{0} & \frac{\rho h^{3}}{12} \mathbf{N}_{\Theta}^{\mathbf{T}} \mathbf{N}_{\Theta}
\end{array}\right] d \Omega^{e}
\end{aligned}
$$

where $\Omega^{e}$ is the domain of element $e$. The matrices in Eq. (11) and (12) are found by summation of the element matrices, that is

$$
\mathbf{K}_{0}=\sum_{e=1}^{n} \mathbf{k}_{0}^{e}, \quad \mathbf{K}_{1}=\sum_{e=1}^{n} \mathbf{k}_{1}^{e}, \quad \text { etc }
$$

where $n$ is the total number of elements, and $\sum$ should be understood as the finite element assembly operator (see e. g. [20]) 